\title{
The SERPINA4 rs2070777 AA Genotype is Associated with an Increased Risk of Recurrent Miscarriage in a Southern Chinese Population
}

This article was published in the following Dove Press journal: International Journal of Women's Health

\author{
Di Che ${ }^{1, *}$ \\ Zhenzhen Fang ${ }^{2, *}$ \\ Lei $\mathrm{Pi}^{\mathrm{l}}$ \\ Yufen $X u^{\prime}$ \\ LanYan Fu' \\ Huazhong Zhou' \\ Xiaoqiong $\mathrm{Gu}^{1,3,4}$
}

'Department of Clinical Biological Resource Bank, Guangzhou Institute of Pediatrics, Guangzhou Women and Children's Medical Center, Guangzhou Medical University, Guangzhou, People's Republic of China; ${ }^{2}$ Program of Molecular Medicine, Guangzhou Women and Children's Hospital, Zhongshan School of Medicine, Sun Yat-Sen University, Guangzhou, People's Republic of China;

${ }^{3}$ Department of Clinical Lab, Guangzhou Women and Children's Medical Center, Guangzhou Medical University, Guangzhou, People's Republic of China: ${ }^{4}$ Department of Blood Transfusion, Guangzhou Women and Children's Medical Center, Guangzhou Medical University, Guangzhou, People's Republic of China

*These authors contributed equally to this work
Background: Many inflammation-related gene polymorphisms are associated with susceptibility to recurrent miscarriage. SERPINA4 is involved in inflammation and is associated with susceptibility to a variety of diseases, but its relevance in recurrent miscarriage is unclear. Therefore, this study aimed to investigate the relationship between SERPINA4 gene polymorphisms and susceptibility to recurrent spontaneous abortion.

Methods: Two SERPINA4 polymorphisms were genotyped in 631 patients with recurrent miscarriage and 771 controls by TaqMan real-time polymerase chain reaction, and the strength of each association was evaluated through 95\% confidence intervals (CIs) and odds ratios (ORs).

Results: The results showed that SERPINA4 rs2070777 AA genotypes were associated with an increased risk of recurrent miscarriage (AA vs AT/TT adjusted OR=1.409, 95\% $\mathrm{CI}=1.032-1.924, \mathrm{P}=0.0309)$, and we also found a significant association between the rs910352 T allele in the SERPINA4 gene and susceptibility to recurrent miscarriage (CT vs $\mathrm{CC}$ adjusted $\mathrm{OR}=1.579,95 \% \mathrm{CI}=1.252-1.992, \mathrm{P}=0.0001$; TT vs $\mathrm{CC}$ adjusted $\mathrm{OR}=1.524$, $95 \% \mathrm{CI}=1.134-2.049, \mathrm{P}=0.0052$ ). The combined analysis of two SNPs of the SERPINA4 gene revealed that carriers with one to two unfavorable genotypes were associated with a higher risk for recurrent miscarriage compared with individuals with no unfavorable genotypes (adjusted $\mathrm{OR}=1.257,95 \% \mathrm{CI}=1.019-1.550$ ). Moreover, our study indicates that having one to two unfavorable genotypes is associated with an increased risk of recurrent miscarriage in women 35-40 years of age.

Conclusion: Our study suggests that SERPINA4 rs2070777AA genotypes might contribute to an increased risk of recurrent miscarriage in a southern Chinese population.

Keywords: recurrent miscarriage, SERPINA4, genetic susceptibility, polymorphism

\section{Introduction}

Recurrent miscarriage is a common complication of pregnancy that is defined as two or more consecutive pregnancy losses before 20 weeks of gestation. ${ }^{1}$ Epidemiological studies have shown that approximately $1-2 \%$ of women are affected by recurrent miscarriage, and this complication places considerable mental and psychological burdens on the patients and their family members. ${ }^{2}$ Many factors contribute to the occurrence of recurrent miscarriage, and these include autoimmune, infectious, endocrine, anatomic and genetic factors. ${ }^{1,2}$ However, the causes of recurrent miscarriages in approximately $50 \%$ of patients are unclear. ${ }^{3}$ The etiopathogenesis of recurrent miscarriage is uncertain, and increasing numbers of
Correspondence: Xiaoqiong Gu Department of Clinical Biological Resource Bank, Guangzhou Institute of Pediatrics, Guangzhou Women and Children's Medical Center, Guangzhou Medical University, 9 Jinsui Road, Guangzhou, Guangdong 510623, People's Republic of China

$\mathrm{Tel} / \mathrm{Fax}+86-20-3807656 \mathrm{I}$

Email guxiaoqiong@gwcmc.org
International Journal of Women's Health 2021:13 I|I-II7

I I I

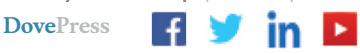


recent studies have suggested that genetic variants might participate in the onset of recurrent miscarriage. ${ }^{4}$ Infective and immunogenetic factors are thought to contribute to the etiopathology of recurrent miscarriage. ${ }^{5,6}$ Many inflammation-related gene polymorphisms, such as FOXP3, $C L O C K, I L-10, T N F-\alpha$ and $I L-17 F$, have been associated with recurrent miscarriage. ${ }^{5,7-9} C R P$ variants are associated with recurrent miscarriage and influence the circulating C-reactive protein levels in chronic inflammatory conditions. ${ }^{10}$ VEGFA polymorphisms are associated with susceptibility to recurrent miscarriage and are independently associated with the VEGF serum levels, and $V E G F A$ single-nucleotide polymorphisms (SNPs) might contribute to the pathogenesis of recurrent miscarriage. ${ }^{11}$

Kallistatin is a member of the serine proteinase inhibitor (SERPIN) superfamily that is encoded by the SERPINA4 gene in humans. ${ }^{12}$ Recent studies have revealed that kallistatin participates in a variety of pathophysiological processes, including the regulation of cancer development, angiogenesis, apoptosis, oxidative stress and inflammation. ${ }^{13-16}$ For example, Lin WC et al reported that kallistatin might inhibit inflammation and apoptosis to protect against sepsis-related acute lung injury. ${ }^{17}$ Moreover, kallistatin blocks the VEGF signaling pathway to inhibit angiogenesis. ${ }^{18} \mathrm{Li} \mathrm{P}$ et al reported that kallistatin regulates miR-203, miR-21 and miR-34a synthesis in breast cancer cells to result in autophagy and apoptosis. ${ }^{19}$ Li D et al suggested that miR-34a is associated with unexplained recurrent spontaneous abortion, ${ }^{20}$ and Magdoud K et al found that plasminogen activator inhibitor type 1 (belonging to the SERPIN family) is associated with an increased risk of recurrent miscarriage. ${ }^{21}$

The above-mentioned studies suggest that SERPINA4 gene polymorphisms might be associated with abortion. However, few studies have investigated the association between SERPINA4 gene polymorphisms and susceptibility to recurrent miscarriage. In this study, we examined the association between susceptibility to recurrent miscarriage and SERPINA4 gene polymorphisms in a southern Chinese population comprising 631 women with recurrent miscarriage and 771 healthy controls with no history of miscarriages.

\section{Materials and Methods}

\section{Study Population}

For this study, 771 healthy controls and 631 women diagnosed with recurrent miscarriage were recruited from
Guangzhou Women and Children's Medical Center (Department of Gynecology) between June 2017 and June 2019. The control women had undergone at least two normal pregnancies and had no history of miscarriage. Recurrent miscarriage was defined as two or more unknown etiologies of spontaneous miscarriages (5-20 weeks of gestation). Specimens were collected from the patients and controls during routine blood tests performed at the hospital. None of the patients with recurrent miscarriage or the control women had a history of uterine anatomic abnormalities or embryo chromosomal abnormalities. Accordance with the Declaration of Helsinki, this study was approved by the Ethics Committee of Guangzhou Women and Children's Medical Center (2018022202). Each participant (patients with recurrent miscarriage and controls) provided written informed consent to participate in the study.

\section{Genotyping and DNA Extraction}

Total genomic DNA was extracted from peripheral blood samples collected from participants using the Blood DNA Kit (Tiangen, Beijing, China) following the manufacturer's specifications. The typing probes (rs910352, [C_9596926_10] and rs2070777 [C_15867824_20]) were purchased from Applied Biosystems (Applied Biosystems TaqMan, Foster City, CA, USA). Genotyping for the two SNPs (rs910352 and rs2070777) was performed in a 384well plate with an ABI Q6 instrument according to the TaqMan real-time polymerase chain reaction protocol (Applied Biosystems TaqMan, Foster City, CA, USA). PCR amplification was performed in a final volume of 5 $\mu \mathrm{L}$, which consisted of $0.04 \mu \mathrm{L}$ of the primers, $2.5 \mu \mathrm{L}$ of $2 \times$ Mix (Tiangen, Beijing, China), $1.46 \mu \mathrm{L}$ of ddH2O and 2.5 ng of DNA.

\section{Statistical Analysis}

The genotypic and demographic differences between the control subjects and the patients with recurrent miscarriage were tested using the two-sided chi-square test. HardyWeinberg equilibrium (HWE) was tested by the goodnessof-fit $\chi 2$ test. The association between the SERPINA4 gene polymorphism and susceptibility to recurrent miscarriage was described by $95 \%$ confidence intervals (CIs) and odds ratios (ORs). A stratified analysis was performed with respect to age and the number of abortions. All statistical analyses were performed using SAS software (version 9.4; SAS Institute, Cary, NC, USA). P $<0.05$ was considered to indicate statistical significance. 


\section{Results}

\section{Population Characteristics}

A total of 631 patients with recurrent miscarriage and 771 controls were included in this study (Table 1). No significant difference in age was found between the patients with recurrent miscarriage and the controls $(32.39 \pm 5.38$ vs $32.50 \pm 5.20$ years old, $\mathrm{P}=0.6978$ ). Moreover, among the patients with recurrent miscarriage, approximately $59.59 \%$ had suffered two to three spontaneous abortions, and $40.41 \%$ suffered at least four spontaneous abortions.

\section{Relationship Between SERPINA4 Polymorphisms and Susceptibility to Recurrent Miscarriage}

We detected the genotype frequency distributions of the controls and patients with recurrent miscarriage to study the association between SERPINA4 polymorphisms and susceptibility to recurrent miscarriage (as shown in Table 2). No significant deviations from $\mathrm{HWE}$ were detected in the control group ( $\mathrm{P}=0.173$ for rs910352, $\mathrm{P}=0.226$ for rs2070777). After adjustments for age, the rs2070777 AA genotype was associated with a significantly increased risk of recurrent miscarriage compared with the TT/AT genotypes (TA vs TT: adjusted $\mathrm{OR}=1.437,95 \% \mathrm{CI}=1.151-1.794, \mathrm{P}=0.0014$; AA vs TT: adjusted $\mathrm{OR}=1.909,95 \% \mathrm{CI}=1.369-2.661, \mathrm{P}=0.0001$; AA vs TT/AT adjusted $\mathrm{OR}=1.409,95 \% \mathrm{CI}=1.032-1.924$, $\mathrm{P}=0.0309$ ). In addition, we found a significant association between the rs910352 T allele in the SERPINA4 gene and susceptibility to recurrent miscarriage (CT vs CC: adjusted
$\mathrm{OR}=1.579,95 \% \mathrm{CI}=1.252-1.992, \mathrm{P}=0.0001$; TT vs $\mathrm{CC}$ : adjusted $\mathrm{OR}=1.524, \quad 95 \% \quad \mathrm{CI}=1.134-2.049, \quad \mathrm{P}=0.0052)$. Consequently, the risk genotypes used for the calculation were SERPINA4 rs910352 CT/TT and 2070777 AA. Compared with individuals with no unfavorable genotype, those who carried one or two unfavorable genotypes exhibited an increased risk for recurrent miscarriage (adjusted $\mathrm{OR}=1.257,95 \% \mathrm{CI}=1.019-1.550$ ).

\section{Stratification Analysis}

We further explored the associations between the SERPINA4 gene variant genotypes of the two selected SNPs (rs910352 and rs2070777) and the risk of recurrent miscarriage through a stratified analysis based on the number of abortions and age (Table 3). Compared with the rs2070777 AA genotype, the AT/TT genotypes were associated with a higher risk for women with two to three miscarriages (adjusted $\mathrm{OR}=1.548,95 \% \mathrm{CI}=1.088-2.201$, $\mathrm{P}=0.0151$ ) due to the cumulative effect of gene mutations (microeffect gene accumulators can exert a significant phenotypic effect). We combined the analysis of rs $910352 \mathrm{CT} /$ TT and rs2070777 AA with respect to risk for recurrent abortion. The combined analysis for all risk genotypes among individuals aged 35-40 years revealed that individuals carrying one or two risk genotypes had a higher risk than individuals with no risk genotypes (adjusted $\mathrm{OR}=1.571,95 \% \mathrm{CI}=1.135-2.175$ ). However, no significant associations were observed in the other stratified analyses, such as those conducted with the group of individuals with four miscarriages.

Table I Frequency Distribution of Selected Characteristics in Patients with Recurrent Miscarriage and Controls

\begin{tabular}{|c|c|c|c|c|c|}
\hline \multirow[t]{2}{*}{ Variables } & \multicolumn{2}{|c|}{ Cases $(n=631)$} & \multicolumn{2}{|c|}{ Controls $(n=77 I)$} & \multirow[t]{2}{*}{$\mathbf{P}^{\mathbf{a}}$} \\
\hline & No. & $\%$ & No. & $\%$ & \\
\hline Age range, years & $20-46$ & & $20-49$ & & \\
\hline Mean \pm SD & $32.39 \pm 5.38$ & & $32.50 \pm 5.20$ & & 0.6978 \\
\hline$<35$ & 419 & 66.4 & 516 & 66.75 & \\
\hline $35-40$ & 155 & 24.56 & 187 & 24.19 & \\
\hline$>40$ & 57 & 9.03 & 70 & 9.06 & \\
\hline \multicolumn{6}{|c|}{ No. and $\%$ of abortions } \\
\hline $2-3$ & 376 & 59.59 & & & \\
\hline$\geq 4$ & 255 & 40.41 & & & \\
\hline
\end{tabular}

Notes: ${ }^{\text {a }}$ The $\mathrm{P}$ value was obtained from a two-sided $\chi 2$ test of the distributions between the patients with recurrent miscarriage and the controls. 
Table 2 Genotype and Allele Frequencies of SERPINA4 in Patients with Recurrent Miscarriage and Controls

\begin{tabular}{|c|c|c|c|c|c|c|c|}
\hline Genotype/Allele & $\begin{array}{l}R M \\
(N=63 I)\end{array}$ & $\begin{array}{l}\text { Controls } \\
(\mathrm{N}=77 \mathrm{I})\end{array}$ & P-value & OR (95\% Cl) & P-value & $\begin{array}{l}\text { Adjusted OR } \\
(95 \% \mathrm{Cl})\end{array}$ & P-value ${ }^{b}$ \\
\hline \multicolumn{8}{|c|}{ SERPINA4/rs910352 C > T $($ HWE $=0.173)$} \\
\hline $\mathrm{CC}$ & 194(30.74) & $266(34.4 I)$ & 0.3388 & 1.00 & I & 1.00 & l \\
\hline $\mathrm{CT}$ & $312(49.45)$ & $359(46.44)$ & l & $1.577(1.251-1.988)$ & 0.0001 & $1.579(1.252-1.992)$ & 0.0001 \\
\hline TT & $125(19.81)$ & $148(19.15)$ & l & $1.532(1.140-2.060)$ & 0.0047 & $1.524(1.134-2.049)$ & 0.0052 \\
\hline Dominant (TT/TC vs CC) & $437(69.26)$ & $507(65.59)$ & 0.1449 & $1.182(0.944-1.480)$ & 0.1455 & I.I86(0.947-I.485) & 0.1382 \\
\hline Recessive (TT vs CT/AA) & $506(80.19)$ & $625(80.85)$ & 0.7547 & $1.043(0.800-1.360)$ & 0.7543 & $1.039(0.797-1.355)$ & 0.777 \\
\hline \multicolumn{8}{|c|}{ SERPINA4//rs2070777 T>A (HWE = 0.226) } \\
\hline $\mathrm{TT}$ & $233(36.93)$ & $319(41.27)$ & 0.0541 & 1.00 & I & 1.00 & l \\
\hline TA & $302(47.86)$ & $367(47.48)$ & l & $1.430(1.146-1.785)$ & 0.0016 & $1.437(1.151-1.794)$ & 0.0014 \\
\hline AA & $96(15.21)$ & $87(11.25)$ & I & $1.918(1.376-2.673)$ & 0.0001 & $1.909(1.369-2.661)$ & 0.0001 \\
\hline Dominant (AA/TA vs TT) & $398(63.07)$ & $454(58.73)$ & 0.0972 & $1.200(0.967-1.490)$ & 0.0977 & $1.206(0.97 \mid-1.497)$ & 0.0896 \\
\hline Recessive (AA vs TA/TT) & $535(84.79)$ & $686(88.75)$ & 0.0288 & $1.415(1.036-1.932)$ & 0.0289 & $1.409(1.032-1.924)$ & 0.0309 \\
\hline \multicolumn{8}{|c|}{ Combined risk-effect of genotypes* } \\
\hline 0 & $346(54.83)$ & $285(45.17)$ & & 1 & l & I & l \\
\hline $1-2$ & $517(60.19)$ & $342(39.8 I)$ & 0.0388 & $1.264(1.025-1.558)$ & 0.0283 & $1.257(1.019-1.550)$ & 0.0326 \\
\hline
\end{tabular}

Notes: *The risk genotypes used for the calculation were rs910352 CT/TT + rs2070777 AA. Dominant: homozygous rare+heterozygous vs homozygous frequent allele. Recessive: homozygous rare vs heterozygous +homozygous frequent allele. Statistically significant values are shown in bold (P<0.05). ${ }^{b} A d j u s t e d$ for age.

Abbreviations: OR, odds ratio; HWE, Hardy-Weinberg equation; RM, recurrent miscarriage.

\section{Discussion}

This case-control study revealed that the SERPINA4 rs2070777 AA variant contributed to an increased risk of recurrent miscarriage in a southern Chinese population, and the increased risk in patients who carry one or two risk genotypes was more obvious in women aged 35-40 years. To our knowledge, this study constitutes the first investigation of the association between susceptibility to recurrent miscarriage and SERPINA4 polymorphisms in a southern Chinese population.

The human kallistatin gene (SERPINA4) is located on $14 \mathrm{q} 32.13$ and encodes kallistatin, a protein involved in the regulation of a variety of biological processes. ${ }^{22}$ The SNPs rs910352 and rs2070777 are located in an intronic region of the SERPINA4 gene. SERPINA4 is involved in regulating the expression of multiple genes and participates in the activation of multiple signaling pathways, including angiogenesis, vasodilation, anti-inflammation, apoptosis, antioxidant, and fibrosis. ${ }^{23-25}$ Recent studies have found that SERPINA4 gene polymorphisms are involved in the disease process. Vilander LM et al found that the SERPINA4
SNP rs2093266 contributes to the development of severe acute kidney injury. ${ }^{26}$ PAI-1 belongs to the SERPIN family, which contributes to an increased risk of recurrent miscarriage. $^{21}$ Tamar Madar et al found that low levels of circulating alpha-1 antitrypsin, which belongs to the SERPIN family, are associated with spontaneous abortion. ${ }^{27}$ However, whether SERPINA4 polymorphisms are related to abortion has not been investigated. In our study, we evaluated the associations between SERPINA4 gene (rs910352 C > T and rs2070777 T>A) polymorphisms and susceptibility to recurrent miscarriage in 631 patients and 771 healthy controls. Our results showed that the rs2070777 AA variant is a risk factor for recurrent miscarriage susceptibility. To our knowledge, this study provides the first assessment of the association of SERPINA4 polymorphisms with recurrent miscarriage risk in a southern Chinese population. We propose that the rs28270177 AA variant might play an important role in the pathogenesis of patients with recurrent miscarriage.

Many studies have suggested that abortion is related to age and that advanced maternal age is related to an increasing 


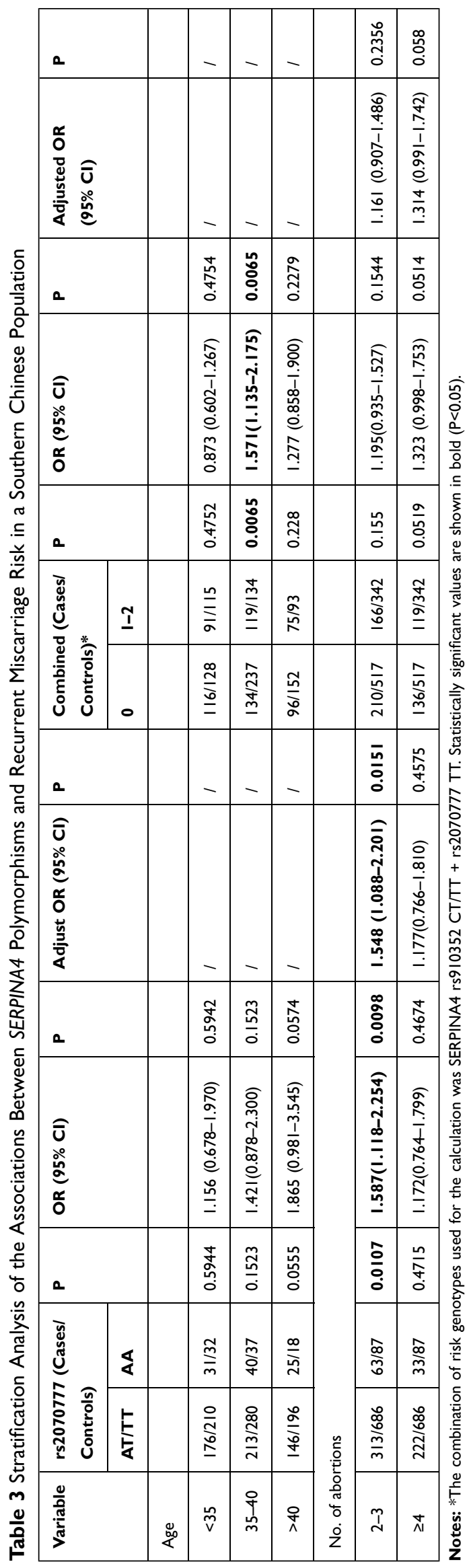

incidence of recurrent miscarriage. ${ }^{28}$ Embryonic chromosomal abnormalities are the most common cause of early miscarriage, and the proportion of chromosomal abnormalities increases as the maternal age increases ( $\geq 35$ years). ${ }^{29}$ Studies have also found that among pregnant women older than 30 years, the risk of miscarriage increases with maternal age. ${ }^{30}$ Nybo Andersen AM et al found that women $\leq 35$ years have a higher risk of miscarriage in the range of 9 to $12 \%$ and that this risk increases to $75 \%$ in women older than 40 years. ${ }^{31}$ Although our study population excluded embryonic chromosomal abnormalities, the combined analysis for all risk genotypes among individuals aged 35-40 years revealed that individuals carrying one or two risk genotypes had a higher risk than individuals with no risk genotypes. Moreover, many studies have found that women with previous abortions have an increased risk of abortion. ${ }^{32,33}$ Interestingly, our research found that SERPINA4 rs2070777 AA genotypes are associated with a significantly increased risk among those with two to three miscarriages. However, the increased risk associated with the number of miscarriages was not observed in the group with more than four miscarriages, which might be related to the multiple biological functions of kallistatin. The reason for this finding is worth further exploration. Further studies with a larger sample size are needed to confirm the results.

The limitations of this study should be noted. First, only rs910352 $\mathrm{C}>\mathrm{T}$ and $\mathrm{rs} 2070777 \mathrm{~T}>\mathrm{A}$ were included in our study, and other gene polymorphisms were not covered. Second, the sample size in the current study was relatively small, and larger sample sizes are needed to confirm the relationship between susceptibility to recurrent miscarriage and SERPINA4 gene polymorphisms. Third, due to the retrospective design of the study, only geographical factors and age were collected in this study. Other factors, such as eating habits and environmental exposure, were not collected. Moreover, the rs910352 heterozygous risk genotype $\mathrm{CT}$ in the SERPINA4 gene is associated with a higher risk of OR than homozygous TT. This finding might suggest that this rs910352 TT genotype is not as important for recurrent miscarriage susceptibility. Therefore, the study of the correlation between SERPINA4 rs2070777AA genotypes and recurrent miscarriage needs to be further expanded to verify the research findings.

In summary, our study suggests that the SERPINA4 rs2070777 AA variant is associated with increased susceptibility to recurrent miscarriage in a southern Chinese population. However, studies with a larger sample size and practical studies should be conducted to further explore the 
roles of the SERPINA4 gene in susceptibility to recurrent miscarriage.

\section{Data Sharing Statement}

Please contact the Correspondence author for data requests.

\section{Acknowledgments}

The authors would like to thank the Clinical Biological Resource Bank of Guangzhou Women and Children's Medical Center for providing all of the clinical samples. This study was funded by the Guangdong Natural Science Foundation (China, 2019A1515012061), Guangzhou Science and Technology Program Key Projects (China, 201904010486 and 201804010035), Guangzhou Institute of Pediatrics/Guangzhou Women and Children's Medical Center Fund (China, grant numbers GCP-2019-006, YIP2018-019, YIP-2019-050, and GCP-2019-003) and Guangzhou Medical and Health Technology Projects (China, 20191A011033 and 20191A011021).

\section{Author Contributions}

All the authors contributed significantly to this work and support the publication of the manuscript. All authors contributed to data analysis, drafting or revising the article, have agreed on the journal to which the article will be submitted, gave final approval of the version to be published, and agree to be accountable for all aspects of the work.

\section{Disclosure}

The authors report no conflicts of interest.

\section{References}

1. Rai R, Regan L. Recurrent miscarriage. Lancet. 2006;368 (9535):601-611. doi:10.1016/S0140-6736(06)69204-0

2. Ford HB, Schust DJ. Recurrent pregnancy loss: etiology, diagnosis, and therapy. Rev Obstet Gynecol. 2009;2:76-83.

3. Toth B, Jeschke U, Rogenhofer N, Scholz C, Wurfel W. Thaler CJ and Makrigiannakis A. Recurrent miscarriage: current concepts in diagnosis and treatment. $J$ Reprod Immunol. 2010;85(1):25-32. doi:10.1016/ j.jri.2009.12.006

4. Daher S, Mattar R, Gueuvoghlanian-Silva BY, Torloni MR. Gueuvoghlanian-Silva BY and Torloni MR. Genetic polymorphisms and recurrent spontaneous abortions: an overview of current knowledge. Am $j$ Reproduct Immunology. 2012;67(4):341-347. doi:10.1111/j.1600-0897.2012.01123.x

5. Muyayalo KP, Li ZH, Mor G, Liao A-H. Mor G and Liao AH. Modulatory effect of intravenous immunoglobulin on Th17/Treg cell balance in women with unexplained recurrent spontaneous abortion. Am j Reproduct Immunology. 2018;80(4):e13018. doi:10.1111/ aji.13018

6. Grimstad F, Krieg S. Immunogenetic contributions to recurrent pregnancy loss. J Assist Reprod Genet. 2016;33(7):833-847. doi:10.1007/ s10815-016-0720-6
7. Najafi S, Hadinedoushan H, Eslami G, Aflatoonian A. Eslami G and Aflatoonian A. Association of IL-17A and IL-17 F gene polymorphisms with recurrent pregnancy loss in Iranian women. $J$ Assist Reprod Genet. 2014;31(11):1491-1496. doi:10.1007/s10815-014-0294-0

8. Ali Rahmani S. Paknejad Z and Mohammadkhanlou M. The effects of tumor necrosis factor-alpha (TNF-alpha) and IL-1 receptor antagonist (IL-1Ra) polymorphisms on recurrent abortion in Azari women. Ginekol Pol. 2017;88:421-427.

9. Bahadori M, Zarei S, Zarnani AH, et al. 6, IL-10 and IL-17 gene polymorphisms in Iranian women with recurrent miscarriage. Iranian j Immunology. 2014;11:97-104.

10. Ahmed SK, Mahmood N, Malalla ZH, Alsobyani FM, Al-Kiyumi IS, Almawi WY. Al-Kiyumi IS and Almawi WY. C-reactive protein gene variants associated with recurrent pregnancy loss independent of CRP serum levels: a case-control study. Gene. 2015;569(1):136-140. doi:10.1016/j.gene.2015.05.052

11. Almawi WY, Saldanha FL, Mahmood NA, Al-Zaman I, Sater MS, Mustafa FE. Sater MS and Mustafa FE. Relationship between VEGFA polymorphisms and serum VEGF protein levels and recurrent spontaneous miscarriage. Human Reproduction. 2013;28 (10):2628-2635. doi:10.1093/humrep/det308

12. Chao J, Schmaier A, Chen LM, Yang Z, Chao L. Yang Z and Chao L. Kallistatin, a novel human tissue kallikrein inhibitor: levels in body fluids, blood cells, and tissues in health and disease. $J$ Lab Clin Med. 1996;127(6):612-620. doi:10.1016/S0022-2143(96) 90152-3

13. Chao J, Li P, Chao L. Li P and Chao L. Kallistatin suppresses cancer development by multi-factorial actions. Crit Rev Oncol Hematol. 2017;113:71-78. doi:10.1016/j.critrevonc.2017.03.011

14. Chao J, Li P, Chao L. Li P and Chao L. Kallistatin: double-edged role in angiogenesis, apoptosis and oxidative stress. Biol Chem. 2017;398 (12):1309-1317. doi:10.1515/hsz-2017-0180

15. Li J. Krishna SM and Golledge J. The Potential Role of Kallistatin in the Development of Abdominal Aortic Aneurysm. Int $J$ Mol Sci. 2016;17

16. Chao J, Miao RQ, Chen V, Chen L-M, Chao L. Chen LM and Chao L. Novel roles of kallistatin, a specific tissue kallikrein inhibitor, in vascular remodeling. Biol Chem. 2001;382(1):15-21. doi:10.1515/ BC.2001.003

17. Lin WC, Chen CW, Huang YW, Chao L, Chao J. Lin YS and Lin CF. Kallistatin protects against sepsis-related acute lung injury via inhibiting inflammation and apoptosis. Sci Rep. 2015;5:12463. doi: $10.1038 /$ srep 12463

18. Huang KF, Yang HY, Xing YM, Lin JS, Diao Y. Lin JS and Diao Y. Recombinant human kallistatin inhibits angiogenesis by blocking VEGF signaling pathway. $J$ Cell Biochem. 2014;115(3):575-584. doi: $10.1002 /$ jcb. 24693

19. Li P, Guo Y, Bledsoe G, Yang Z, Chao L, Chao J. Chao L and Chao J. Kallistatin induces breast cancer cell apoptosis and autophagy by modulating Wnt signaling and microRNA synthesis. Exp Cell Res. 2016;340(2):305-314. doi:10.1016/j.yexcr.2016.01.004

20. Li D, Li J. Association of miR-34a-3p/5p, miR-141-3p/5p, and miR-24 in Decidual Natural Killer Cells with Unexplained Recurrent Spontaneous Abortion. Med Sci Monitor. 2016;22:922-929. doi:10. 12659/MSM.895459

21. Magdoud K, Herbepin VG, Touraine R. Almawi WY and Mahjoub T. Plasminogen activator inhibitor $14 \mathrm{G} / 5 \mathrm{G}$ and $-844 \mathrm{G} / \mathrm{A}$ variants in idiopathic recurrent pregnancy loss. Am $j$ Reproduct Immunology. 2013;70:246-252. doi:10.1111/aji.12116

22. Chao J, Bledsoe G, Chao L. Bledsoe G and Chao L. Protective Role of Kallistatin in Vascular and Organ Injury. Hypertension. 2016;68 (3):533-541. doi:10.1161/HYPERTENSIONAHA.116.07861

23. Chen VC. Chao L and Chao J. Reactive-site specificity of human kallistatin toward tissue kallikrein probed by site-directed mutagenesis. Biochim Biophys Acta. 2000;1479:237-246. doi:10.1016/S01674838(00)00044-3 
24. Chen VC, Chao L, Chao J. Chao L and Chao J. Roles of the P1, P2, and $\mathrm{P} 3$ residues in determining inhibitory specificity of kallistatin toward human tissue kallikrein. $J$ Biol Chem. 2000;275 (49):38457-38466. doi:10.1074/jbc.M005605200

25. Chen VC, Chao L, Pimenta DC, Bledsoe G, Juliano L, Chao J. Juliano $\mathrm{L}$ and Chao J. Identification of a major heparin-binding site in kallistatin. J Biol Chem. 2001;276(2):1276-1284. doi:10.1074/jbc. M005791200

26. Vilander LM, Kaunisto MA, Vaara ST, Pettilä V. Genetic variants in SERPINA4 and SERPINA5, but not BCL2 and SIK3 are associated with acute kidney injury in critically ill patients with septic shock. Critical Care. 2017;21(1):47. doi:10.1186/s13054-017-1631-3

27. Madar T, Shahaf G, Sheiner E, et al. Holcberg G and Lewis EC. Low levels of circulating alpha-1 antitrypsin are associated with spontaneous abortions. J Matern Fetal Neonatal Med. 2013;26 (18):1782-1787. doi:10.3109/14767058.2013.801955

28. Dean DD, Agarwal S, Tripathi P. Agarwal S and Tripathi P. Connecting links between genetic factors defining ovarian reserve and recurrent miscarriages. J Assist Reprod Genet. 2018;35 (12):2121-2128. doi:10.1007/s10815-018-1305-3
29. Yuan SM, Liao C, Li DZ, Huang JZ, Hu SY, Ke M. Zhong HZ and Y CX. [Chorionic villus cell culture and karyotype analysis in 1983 cases of spontaneous miscarriage]. Zhonghua Fu Chan ke Za Zhi. 2017;52:461-466. doi:10.3760/cma.j.issn.0529-567X.2017.07.006

30. Magnus MC, Wilcox AJ, Morken NH. Weinberg CR and Haberg SE. Role of maternal age and pregnancy history in risk of miscarriage: prospective register based study. BMJ. 2019;364:1869. doi:10.1136/ bmj.1869

31. Nybo Andersen AM, Wohlfahrt J, Christens P. Olsen J and Melbye M. Maternal age and fetal loss: population based register linkage study. BMJ. 2000;320:1708-1712. doi:10.1136/bmj.320.7251.1708

32. Knudsen UB, Hansen V. Juul S and Secher NJ. Prognosis of a new pregnancy following previous spontaneous abortions. Eur J Obstet Gynecol Reprod Biol. 1991;39:31-36. doi:10.1016/0028-2243(91) 90138-B

33. Haas DM. Hathaway TJ and Ramsey PS. Progestogen for preventing miscarriage in women with recurrent miscarriage of unclear etiology. Cochrane Database Sys Rev. 2018;10:CD003511. doi:10.1002/ 14651858.CD003511.pub4
International Journal of Women's Health

\section{Publish your work in this journal}

The International Journal of Women's Health is an international, peerreviewed open-access journal publishing original research, reports, editorials, reviews and commentaries on all aspects of women's healthcare including gynecology, obstetrics, and breast cancer. The
Dovepress

manuscript management system is completely online and includes a very quick and fair peer-review system, which is all easy to use. Visit http://www.dovepress.com/testimonials.php to read real quotes from published authors. 